\title{
Effects of quantitative trait loci determining testicular weight in DDD/Sgn inbred mice are strongly influenced by circulating testosterone levels
}

\author{
Jun-ichi Suto ${ }^{1, *}$ and Misaki Kojima²
}

\author{
* Corresponding Author: Jun-ichi Suto \\ Tel: +81-29-838-6052, Fax: +81-29-838-7106, \\ E-mail: jsuto@affrc.go.jp
}

'Institute of Agrobiological Sciences, National Agriculture and Food Research Organization (NARO), Tsukuba, Ibaraki 305-8634, Japan

2 Institute of Livestock and Grassland Science, National Agriculture and Food Research Organization (NARO), Tsukuba, Ibaraki 305-0901, Japan

ORCID

Jun-ichi Suto

https://orcid.org/0000-0003-0659-2257

Misaki Kojima

https://orcid.org/0000-0002-1921-8160

Submitted Oct 17, 2018; Revised Feb 18, 2019; Accepted Mar 6, 2019
Objective: Testicular growth and development are strongly influenced by androgen. Although both testis weight and plasma testosterone level are inherited traits, the interrelationship between them is not fully established. Males of DDD/Sgn (DDD) mice are known to have extremely heavy testes and very high plasma testosterone level among inbred mouse strains. We dissected the genetic basis of testis weight and analyzed the potential influence of plasma testosterone level in DDD mice.

Methods: Quantitative trait loci (QTL) mapping of testis weight was performed with or without considering the influence of plasma testosterone level in reciprocal $\mathrm{F}_{2}$ intercross populations between DDD and C57BL/6J (B6) mice, thereby assessing the influence of testosterone on the effect of testis weight QTL. Candidate genes for testis weight QTL were investigated by next-generation sequencing analysis.

Results: Four significant QTL were identified on chromosomes 1, 8, 14, and 17. The DDDderived allele was associated with increased testis weight. The $\mathrm{F}_{2}$ mice were then divided into two groups according to the plasma testosterone level $\left(\mathrm{F}_{2}\right.$ mice with relatively "low" and "high" testosterone levels), and QTL scans were again performed. Although QTL on chromosome 1 was shared in both $\mathrm{F}_{2}$ mice, QTL on chromosomes 8 and 17 were identified specifically in $\mathrm{F}_{2}$ mice with relatively high testosterone levels. By whole-exome sequencing analysis, we identified one DDD-specific missense mutation Pro29Ser in alpha tubulin acetyltransferase 1 (Atat1).

Conclusion: Most of the testis weight QTL expressed stronger phenotypic effect when they were placed on circumstance with high testosterone level. High testosterone influenced the QTL by enhancing the effect of DDD-derived allele and diminishing the effects of B6-derived allele. Since Pro29Ser was not identified in other inbred mouse strains, and since Pro29 in Atat 1 has been strongly conserved among mammalian species, Atat 1 is a plausible candidate for testis weight QTL on chromosome 17.

Keywords: DDD/Sgn Mice; Quantitative Trait Loci (QTL) Mapping; Testis Weight; Testosterone Level; Whole-exome Sequence Analysis

\section{INTRODUCTION}

Testis weight is a physiologically important quantitative trait because of its direct implication in male fertility, i.e., spermatogenic ability. Several lines of evidence suggest that sperm production rate depends on testis weight $[1,2]$. Besides spermatogenesis, testis weight might be associated with various physiological processes; e.g., mating and aggression behaviors [3]. Interestingly, when lines were selected for testis weight, a correlated response for ovulation rate in females emerged in mice [4] and golden hamsters [5]. This suggests that sex-limited phenotypes are mutually correlated probably by sharing common physiologic pathways [6]. 
Therefore, elucidating the genetic basis underlying testis weight will be help to reveal various physiologic mechanisms of reproductive processes in males as well as females.

Since testis weight varies widely among inbred mouse strains (pared testis weight ranged about 100 to $300 \mathrm{mg}$ ), several studies pursuing testis weight genes have been performed [3,7-10]. Since males of the inbred mouse DDD/Sgn strain are known to have extremely heavy testes among inbred mouse strains, we have previously performed quantitative trait loci (QTL) mapping analyses in two $\mathrm{F}_{2}$ intercross populations using DDD mice; i.e., $\mathrm{F}_{2}$ mice between DDD and DH/ Sgn (DH) inbred mice [9] and $\mathrm{F}_{2}$ mice between DDD and $\mathrm{CBA} / \mathrm{N}(\mathrm{CBA})$ inbred mice [10]. In $\mathrm{DDD} \times \mathrm{DH} \mathrm{F}_{2}$ mice, we identified significant testis weight QTL on chromosome $(\mathrm{Chr})$ 9 (Twdq1) and suggestive QTL on Chrs 4, 5, 14, 17, and 18. In DDD $\times \mathrm{CBA} \mathrm{F}_{2}$ mice, we identified significant QTL on Chrs $1(T w d q 2)$ and $\mathrm{X}(T w d q 3)$ and suggestive QTL on Chrs 2, 8, 9, 11,13 , and 14 . The localization of QTL differed substantially between two crosses, and if any, there were some overlapping QTL. This finding suggests that there would be additional, yet-to-be-identified genes, which contribute to the heavy testes of DDD mice. In the present study, as a third QTL mapping study aimed at genetically dissecting the heavy testes in DDD mice, we produced and analyzed $\mathrm{F}_{2}$ intercross populations between DDD and C57BL/6J (B6) inbred mice.

In addition to heavy testes, DDD males are known to have very high blood testosterone level $[11,12]$. Indeed, while the average plasma testosterone level in B6 males was $0.49 \pm 0.18$ $\mathrm{ng} / \mathrm{mL}$, that in DDD males was $13.07 \pm 3.12 \mathrm{ng} / \mathrm{mL}$ [12]. Testicular growth and development is strongly influenced by androgen $[13,14]$. Although both testis weight and plasma testosterone level are inherited traits, interrelationship between them is not fully established. We have previously performed QTL mapping for plasma testosterone level in $\mathrm{F}_{2}$ male mice between DDD and B6 [12] but did not detect any significant QTL. Even though the genetic basis of plasma testosterone level in DDD mice is unclear, we hypothesize that high blood testosterone level influence extremely heavy testes in DDD mice. Therefore, in this study, we first performed QTL mapping for testis weight and then investigated the influence of plasma testosterone level on the effect of the testis weight QTL.

We further performed whole-exome sequencing analysis in DDD mice to identify genes underlying testis weight QTL. Since extremely heavy testes are a prominent phenotype of DDD mice among inbred mouse strains, it was expected that there would be candidate genes carrying DDD-specific variations.

\section{MATERIALS AND METHODS}

\section{Animal ethics statement}

All animal procedures were reviewed and approved by the
Institutional Animal Care and Use Committee of the NIAS (authorization number H25-001).

\section{Mice}

The inbred mouse strains DDD and B6 were maintained at the National Institute of Agrobiological Sciences (NIAS, Tsukuba, Japan). Reciprocal crosses between DDD and B6 strains produced $\mathrm{DB}(+\mathrm{DDD} \times \overbrace{}^{\lambda} \mathrm{B} 6) \mathrm{F}_{1}$ and $\mathrm{BD}(+\mathrm{B} 6 \times \overbrace{}^{\lambda} \mathrm{DDD}) \mathrm{F}_{1}$ mice, both of which were intercrossed to produce $\mathrm{DB} \mathrm{F}_{2}$ (n $=150)$ and $\mathrm{BD} \mathrm{F}_{2}(\mathrm{n}=150)$ male mice. Testicular weight was determined in DDD, B6, and $\mathrm{F}_{1}$ mice at the age of 13 to 14 weeks ( $90 \pm 5$ days after birth), and in $\mathrm{F}_{2}$ mice at the age of 11 to 12 weeks ( 71 to 80 days after birth).

All mice were weaned at 4 weeks of age and four to five mice were housed together in each cage during the experiments. All mice were maintained in a specific pathogen-free facility with a regular light cycle, controlled temperature, and humidity. Food (CRF-1; Oriental Yeast Co., Ltd., Tokyo, Japan) and water were freely available throughout the experimental period.

\section{Testosterone and testis weight analyses}

The plasma testosterone level was determined in parental, $\mathrm{F}_{1}$ and $\mathrm{F}_{2}$ mice, as previously described [12]. The paired testes were weighed on an electric balance to the nearest milligram. The weight of the paired testes is simply designated as "testis weight."

\section{Genotyping}

Microsatellite sequence length polymorphisms were identified by electrophoresis after polymerase chain reaction (PCR) amplification of genomic DNA. The PCR amplification was carried out using the Thermal Cycler Dice (TaKaRa Bio Inc., Shiga, Japan). The PCR products were separated on $10 \%$ polyacrylamide gel (Nacalai Tesque Inc., Kyoto, Japan) and were visualized by ethidium bromide (Nacalai Tesque, Japan) staining. In total, 117 microsatellite loci were genotyped. Their chromosomal positions were retrieved from Mouse Genome Informatics (MGI, http://www.jax.org). Because the chromosomal positions of six markers were unavailable, they were determined based on our own linkage map.

\section{Quantitative trait loci analysis}

Normality of testis weight was assessed using the ShapiroWilk W test (JMP13, SAS Institute Japan Inc., Tokyo, Japan). QTL mapping was performed using R/QTL version 1.38-4 $[15,16]$. Single-QTL scans were performed by computing at 1 $\mathrm{cM}$ intervals across the entire genome with or without using the lineage (i.e., the direction of the cross) effect as a covariate. First, we included the lineage effect as an additive covariate in the single-QTL scan, if the lineage effect had a strong effect on testis weight. Next, we included the lineage effect as an in- 
teractive covariate because the effect of QTL may vary with the covariate (i.e., QTL $\times$ covariate interaction). Threshold logarithm of the odds (LOD) scores for significant $(\mathrm{p}<0.05)$ and suggestive $(\mathrm{p}<0.63)$ linkages were determined by performing 1,000 permutations [17,18]. After single-QTL scans, two-QTL scans were performed to identify pairwise interactions. In this case, we strictly adhered to a recommended threshold [15]. Finally, the covariates and the combined effects of all QTL, including those that were significant and suggestive, were assessed using multiple-QTL models [19].

\section{Whole-exome sequence analysis}

To identify nonsynonymous single-nucleotide variation (nsSNV) and/or insertion-deletion in the coding regions of candidate genes, whole-exome sequence analyses were performed. Genomic DNA was extracted from the tail of DDD mice using a genomic DNA purification kit (Wizard Genomic DNA Purification Kit, Promega KK, Tokyo, Japan) and was submitted to Filgen, Inc. (Nagoya, Aichi, Japan) for exome capture and sequencing. Sequence reads were mapped to the mouse reference genome (GRCm38, mm10). Read mapping and variant analyses were performed using CLC Genomics Workbench 7.0.4 and 8.5.1 (Filgen, Japan).

\section{Alpha tubulin acetyltransferase 1 sequencing}

Genomic DNA from inbred strains including DDD, B6, DH/ Sgn, and CBA/N were extracted according the protocol described above. PCR was performed using Atat1-specific primers (F: 5'ttcccgttcgatgtggat and R: 5'-gtaaataacgtgccggttgc). The PCR product was purified by PCR purification kit (LaboPass PCR, Hokkaido System Science., Ltd. Sapporo, Japan) and submitted to Hokkaido System Science for direct sequence with these primers.

\section{Statistical analysis}

Statistical analyses were performed using JMP 13 (SAS Institute, Japan). Testis weight was represented as the mean \pm standard error of the mean (mg). Statistical differences between two groups were analyzed using Student's or Welch's $t$ test. For statistical comparison among more than two groups, the Tukey-Kramer honest significant difference test was used. Statistical significance is defined when $\mathrm{p}$ values are less than 0.05 .

\section{RESULTS}

Localization of testis weight quantitative trait loci Testis weight in DDD males $(\mathrm{n}=28)$ was significantly higher than that in B6 males $(\mathrm{n}=16)(277.6 \pm 3.7 \mathrm{mg}$ vs $206.2 \pm 5.3$ $\mathrm{mg}, \mathrm{p}<0.0001$ ). A clear lineage effect was identified in $\mathrm{F}_{1}$ mice; i.e., testis weight in $\mathrm{BD} \mathrm{F}_{1}$ males $(\mathrm{n}=12)$ was significantly higher than that in $\mathrm{DB} \mathrm{F}_{1}$ males $(\mathrm{n}=15)(301.5 \pm 4.8 \mathrm{mg}$ vs

\section{$279.7 \pm 3.7 \mathrm{mg}, \mathrm{p}<0.002)$.}

Figure 1 depicts a histogram showing the distribution of testis weight in $297 \mathrm{~F}_{2}$ male mice (We failed to determine the testis weight in one $\mathrm{BD} \mathrm{F}_{2}$ and one $\mathrm{DB} \mathrm{F}_{2}$ mouse. In addition, we excluded one $\mathrm{DB} \mathrm{F}_{2}$ mouse from the analysis because the paired testis weight of this mouse was abnormally low; i.e., only $76.8 \mathrm{mg}$. Thus, we phenotyped $297 \mathrm{~F}_{2}$ mice but genotyped $300 \mathrm{~F}_{2}$ mice). The testis weight followed a normal distribution. The average testis weight was $255.8 \pm 1.7 \mathrm{mg}$. Although the difference between $\mathrm{BD} \mathrm{F}_{2}$ and $\mathrm{DB} \mathrm{F}_{2}$ males was not statistically significant $(257.0 \pm 2.3 \mathrm{mg}$ vs $254.6 \pm 2.4 \mathrm{mg}, \mathrm{p}>0.4$ ), we included the lineage effect as an additive covariate in the subsequent single-QTL scan.

A LOD score plot for testis weight in F2 males is shown in Figure 2 (solid lines). As shown in Table 1, four significant QTL were identified on Chr 1@57.5 cM (Twdq2: this locus was coincidental with that identified in $\mathrm{DDD} \times \mathrm{CBA} \mathrm{F}_{2}$ mice [10]; accordingly, the same QTL symbol was assigned), Chr 8@41.5 cM (Twdq4), Chr 14@32.3 cM (Twdq5), and Chr 17@36.1 cM (Twdq6), along with three suggestive QTL on Chr 4@33.1 cM (Twdq7: QTL symbol was assigned to this suggestive QTL because a significant testis weight QTL has been mapped to this region [3]), Chr 7@2.0 cM, and Chr $10 @ 61.5 \mathrm{cM}$ (Twdq8: QTL symbol was assigned to this suggestive QTL because a significant testis weight QTL has been mapped to this region [3]). The DDD allele was associated with increased testis weight at all QTL except for the Twdq8 on Chr 10. We next performed QTL mapping by including

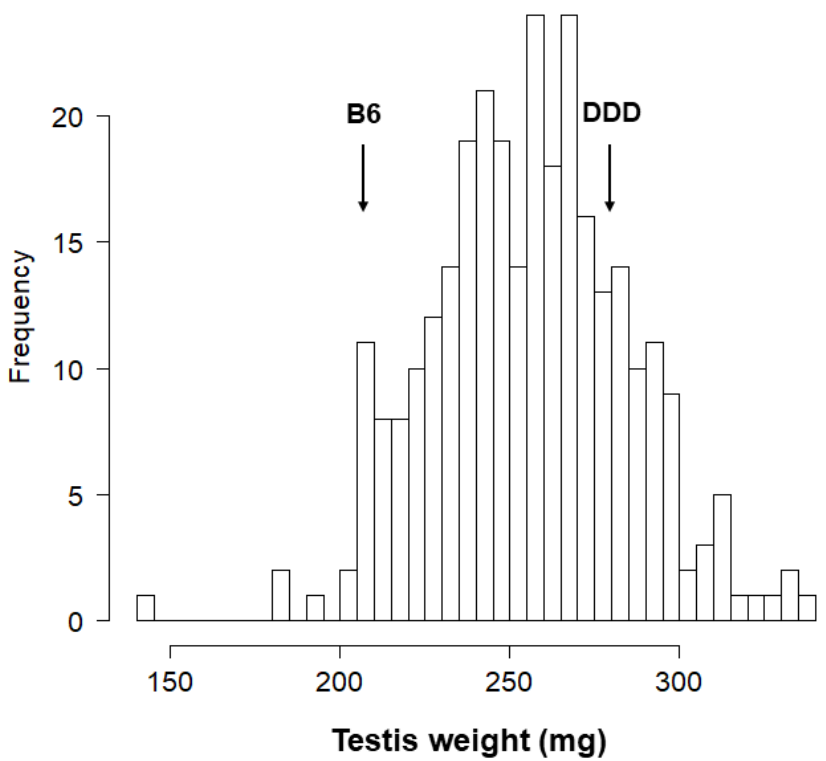

Figure 1. A histogram showing the distribution of testis weight in $297 \mathrm{~F}_{2}$ mice

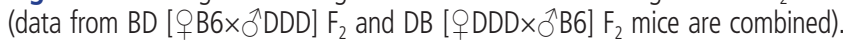
The mean \pm SEM is $255.8 \pm 1.7 \mathrm{mg}$ (The mean \pm SEM testis weight in $B D F_{2}$ mice is $257.0 \pm 2.3 \mathrm{mg}$ and that in $B D F_{2}$ mice is $254.6 \pm 2.4 \mathrm{mg}$. The difference was not statistically significant). The average testis weight of parental $B 6$ and DDD strains are indicated by arrows. SEM, standard error of the mean. 


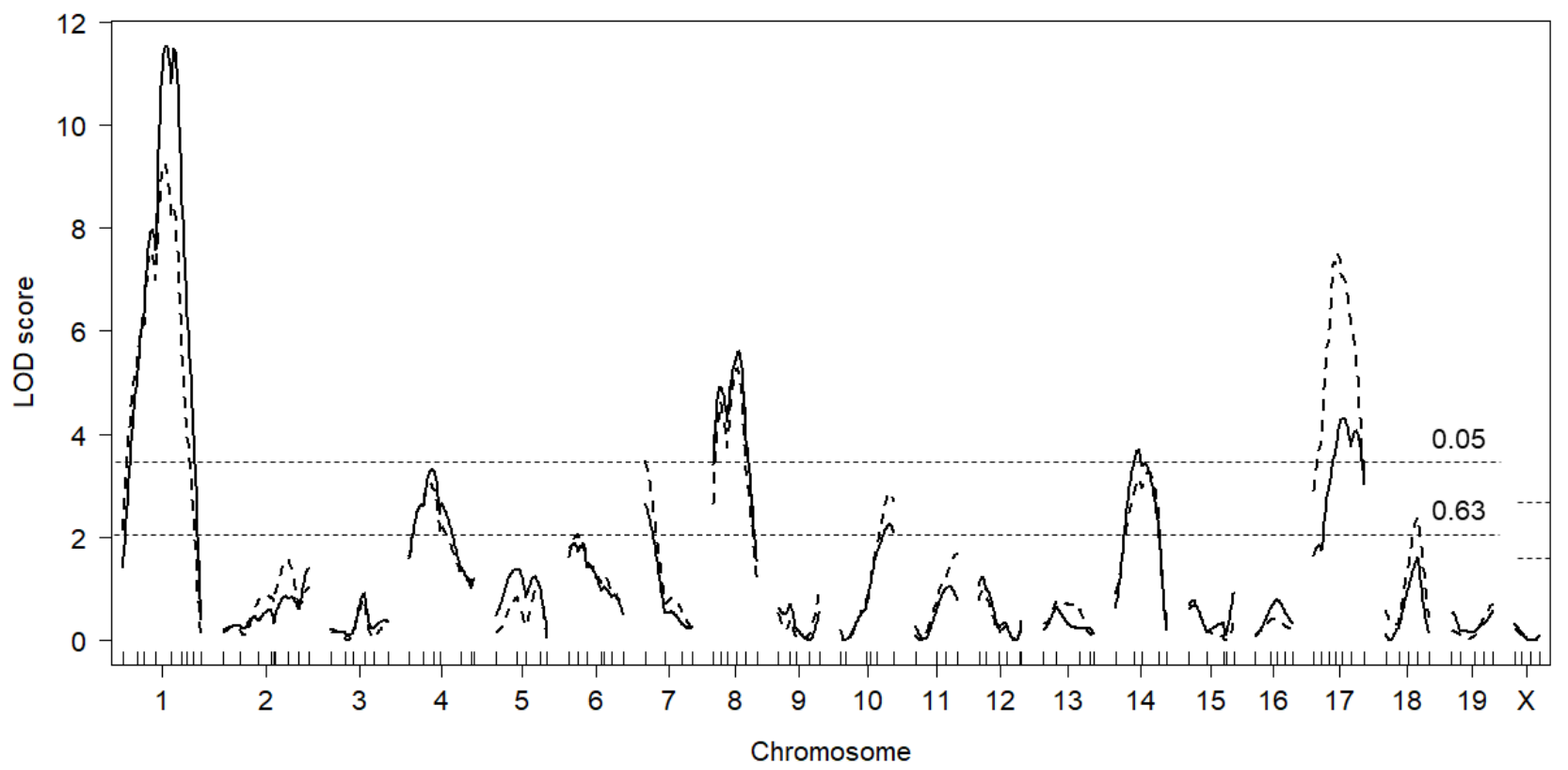

Figure 2. Genome-wide LOD score plot for testis weight when the lineage effect was included as an additive covariate (solid lines) and when the body weight was included as an additive covariate (dashed lines). The $x$-axis represents chromosomal and microsatellite marker positions and the $y$-axis represents LOD scores. Horizontal broken lines indicate the genome-wide threshold LOD score for significant $(p<0.05)$ and suggestive $(p<0.63)$ linkage. Threshold LOD scores for significant and suggestive quantitative trait loci were 3.56 and 2.10 for autosomes and 2.73 and 1.48 for Chr $X$, respectively. LOD, logarithm of the odds.

the lineage effect as an interactive covariate, but found no significant QTLxcovariate interactions (in other words, there were no QTL whose effect differed significantly between BD $\mathrm{F}_{2}$ and DB $\mathrm{F}_{2}$ males). Two-QTL scans with R/QTL revealed a possible pairwise interaction between $\mathrm{Chr} 1$ and $\mathrm{Chr} 8$; however, the LOD score for interaction (lod.int) was substantially lower (2.58) than the recommended threshold value (i.e., 6.3). Multiple regression analysis indicated that the detected QTL explained $41.4 \%$ of the testis weight variation (Table 2).
In the above analysis, we found strong evidence for $T w d q 2$ on Chr 1. Accordingly, we next performed QTL mapping by including the nearest marker (D1Mit102) for Twdq2 as an additive covariate. While the LOD score on Chr 1 shrank to 1.1, the LOD scores for the remaining significant QTL, particularly those for Twdq4 (Chr 8) and Twdq5 (Chr 14), were increased to 6.2 and 4.8 , respectively. In contrast, the LOD score for Twdq6 (Chr 17) was changed subtly (LOD score 4.4) (data not shown). One additional suggestive QTL was

Table 1. Significant and suggestive QTL identified by the genome-wide scan of F2 mice

\begin{tabular}{|c|c|c|c|c|c|c|}
\hline$\overline{Q T L^{1)}}$ & $\mathrm{Chr}$ & Peak cM & $95 \% \mathrm{Cl}^{2)}$ & $\mathrm{LOD}^{3)}$ & Nearest marker & High strain ${ }^{4)}$ \\
\hline$T w d q 2$ & 1 & 57.5 & $49.5-72.5$ & 11.5 & D1Mit102 & DDD \\
\hline \multirow[t]{2}{*}{ Twdq7 } & 4 & 33.1 & $8.1-61.1$ & 3.3 & D4Mit178 & DDD \\
\hline & 7 & 2.0 & $2.0-20.2$ & 2.7 & D7Mit178 & DDD \\
\hline$T w d q 4$ & 8 & 41.5 & $14.5-50.1$ & 5.6 & D8Mit263 & DDD \\
\hline Twdq8 & 10 & 61.5 & $35.5-66.7$ & 2.2 & D10Mit180 & B6 \\
\hline$T w d q 5$ & 14 & 32.3 & $16.3-57.3$ & 3.7 & D14Mit64 & DDD \\
\hline Twdq6 & 17 & 36.1 & $17.1-60.7$ & 4.3 & D17Mit152 & DDD \\
\hline \multicolumn{7}{|c|}{ By including D1Mit102 as an additive covariate } \\
\hline & 5 & 41.6 & $17.8-72.8$ & 2.5 & D5Mit113 & DDD \\
\hline \multicolumn{7}{|c|}{ By including body weight as an additive covariate } \\
\hline \multirow[t]{2}{*}{ Twdq9 } & 7 & 2.0 & $2.0-16.0$ & 3.5 & D7Mit178 & DDD \\
\hline & 18 & 45.9 & $28.9-55.9$ & 2.4 & D18Mit188 & DDD \\
\hline
\end{tabular}

QTL, quantitative trait loci; Cl, confidence interval; LOD, logarithm of the odds.

Lineage effect was included as an additive covariate.

1) QTL symbols were assigned if they were significant, or if they were suggestive but identified as significant at least once previously in different genetic crosses.

2) $95 \% \mathrm{Cl}$ was defined by a $1.5-$ LOD drop.

3) LOD scores for significant QTL are indicated in bold.

${ }^{4)}$ High strain-derived alleles were associated with higher testis weight. 
Table 2. Multiple regression analysis for testis weight

\begin{tabular}{llccc}
\hline Mice & Chr (cM) & df $^{1)}$ & Variance $(\%)^{2)}$ & F value \\
\hline All F $_{2}{ }^{2)}$ & Chr1@57.5 & 2 & 13.7 & 33.0 \\
& Chr4@33.1 & 2 & 2.0 & 4.8 \\
& Chr7@2.0 & 2 & 3.1 & 7.4 \\
& Chr8@41.5 & 2 & 4.9 & 11.8 \\
& Chr10@61.5 & 2 & 1.8 & 4.4 \\
& Chr14@32.3 & 2 & 5.6 & 13.5 \\
& Chr17@37.1 & 2 & 4.5 & 10.7 \\
& Total & 14 & 41.4 & - \\
$\mathrm{F}_{2} \mathrm{~L}$ & Chr1@52.5 & 2 & 12.7 & 10.5 \\
& Chr10@64.5 & 2 & 5.6 & 4.7 \\
& Chr14@53.3 & 2 & 8.3 & 6.9 \\
& Total & 6 & 29.9 & - \\
$\mathrm{F}_{2} \mathrm{H}$ & Chr1@68.5 & 2 & 12.0 & 16.4 \\
& Chr4@33.1 & 2 & 2.7 & 3.7 \\
& Chr8@45.5 & 2 & 5.8 & 8.0 \\
& Chr17@52.1 & 2 & 9.0 & 12.4 \\
& Total & 8 & 39.8 & - \\
\hline
\end{tabular}

\footnotetext{
1) Degrees of freedom.

2) Percentage of total $F_{2}$ phenotypic variance.

${ }^{3)}$ Lineage effect was included as a covariate.
}

identified on Chr 5 (Table 1).

We also performed a single-QTL scan by including body weight as an additive covariate. Since the suggestive QTL on Chr 7 was identified as significant (Table 1), we newly assigned the QTL symbol Twdq9 to this QTL. Clearly, the LOD score for Twdq6 (Chr 17) was substantially increased to 7.7 (Figure 2, dashed lines). One additional suggestive QTL was identified on Chr 18 (Table 1).

\section{Influence of testosterone level on the localization of quantitative trait loci}

Next, we analyzed testis weight by taking circulating testosterone level into consideration, because DDD male has extremely high plasma testosterone level. The $\mathrm{F}_{2}$ mice were then divided into two groups according to the plasma testosterone level; i.e., $\mathrm{F}_{2}$ mice with less than $0.49 \mathrm{ng} / \mathrm{mL}$ testosterone (an average testosterone level in $\mathrm{B} 6$ male) were designated as $\mathrm{F}_{2} \mathrm{~L}\left(\mathrm{~F}_{2}\right.$ with "low" testosterone, $\mathrm{n}=124$, an average and a median testosterone level were $0.33 \pm 0.01 \mathrm{ng} / \mathrm{mL}$ and $0.34 \mathrm{ng} / \mathrm{mL}$, respectively) mice and $\mathrm{F}_{2}$ mice with more than $0.49 \mathrm{ng} / \mathrm{mL}$ testosterone were as $\mathrm{F}_{2} \mathrm{H}\left(\mathrm{F}_{2}\right.$ with "high" testosterone, $\mathrm{n}=176$, an average and a median testosterone level were $7.84 \pm 0.78$ $\mathrm{ng} / \mathrm{mL}$ and $2.02 \mathrm{ng} / \mathrm{mL}$, respectively) mice. The difference in testosterone level between $\mathrm{F}_{2} \mathrm{~L}$ and $\mathrm{F}_{2} \mathrm{H}$ mice was statistically significant $(\mathrm{p}<0.0001)$ when judged by either parametric (Student's $t$-test) or non-parametric (Wilcoxon rank sum test) analysis. On the other hand, there was no significant difference in testis weight in $\mathrm{F}_{2} \mathrm{~L}$ and $\mathrm{F}_{2} \mathrm{H}$ mice $(256.1 \pm 2.6$ $\mathrm{mg}$ vs $255.6 \pm 2.2 \mathrm{mg}, \mathrm{p}>0.8$ ). Single-QTL scans were performed for $\mathrm{F}_{2} \mathrm{~L}$ and $\mathrm{F}_{2} \mathrm{H}$ mice separately. In $\mathrm{F}_{2} \mathrm{~L}$ mice, one significant QTL was identified Chr 1@52.5 cM, along with two suggestive QTL on Chr 10@64.5 cM and Chr 14@53.3 $\mathrm{cM}$ (Table 3; Figure 3). On the other hand, in $\mathrm{F}_{2} \mathrm{H}$ mice, three significant QTL were identified on Chr1@68.5 cM, Chr 8@ $45.5 \mathrm{cM}$, and Chr 17@52.1 cM, along with one suggestive QTL on Chr 4@33.1 cM. Substantial difference in LOD scores between $\mathrm{F}_{2} \mathrm{~L}$ and $\mathrm{F}_{2} \mathrm{H}$ mice was found for QTL on Chrs 1, 4, 8, and 17. Notably, the LOD score for QTL on Chr 17 in $\mathrm{F}_{2} \mathrm{H}$ mice was higher than that identified in all $\mathrm{F}_{2}$ mice (Table 1). Although QTL on Chr 1 explained similar variance in $\mathrm{F}_{2} \mathrm{~L}$ and $\mathrm{F}_{2} \mathrm{H}$ mice, QTL identified in $\mathrm{F}_{2} \mathrm{H}$ mice accounted for slightly more variance compared with those identified in $\mathrm{F}_{2} \mathrm{~L}$ mice (Table 2). Allele effect of significant QTL was investigated in all $\mathrm{F}_{2}, \mathrm{~F}_{2} \mathrm{~L}$, and $\mathrm{F}_{2} \mathrm{H}$ mice (Figure 4). Except for Twdq5 on Chr 14 (Figure 4C), high testosterone level tended to be associated with increasing the effect of the DDDderived allele and with decreasing the effect of the B6-derived allele. Testosterone level appeared not to influence the effect of heterozygotes.

\section{Candidate gene identification for Twdq6 on Chr 17}

By submitting the term "abnormal testis weight" as a query to the MGI database (Mammalian Phenotype Browser), we retrieved 25 genes that were localized within 95\% confidence intervals (CIs) of four significant single-QTL on Chrs 1, 8, 14, and 17 (Table 4). In most cases, testis weight was reduced in the mutant mice of these genes; only 2 of 25 mutants (protein tyrosine phosphatase, receptor type, $\mathrm{V}[\mathrm{Ptprv}]$ and TBC domain family, member 4 [Tbc1d4]) were associated with increased testis weight. Since DDD mice have extremely heavy testis relative to other inbred mice, we assumed that there are DDD-specific genetic variations. To identify such genetic variations, we performed whole-exome sequence analysis. We identified nsSNVs in eight genes, i.e., tudor domain containing 5 ( $T d r d 5)$, neuregulin 1 (Nrg1), nanos C2HC-type zinc finger 3 (Nanos3), synaptonemal complex central element protein 2 (Syce2), Tbc1d4, mutS homolog 5 (Msh5),

Table 3. Significant and suggestive QTL identified by the genome-wide scan of separate $\mathrm{F}_{2}$ mice

\begin{tabular}{lccccc}
\hline Mice & QTL $^{1)}$ & Chr & Peak cM & 95\% Cl & LOD $^{3)}$ \\
\hline $\mathrm{F}_{2} \mathrm{~L}$ & Twdq2 & 1 & 52.5 & $16.5-72.5$ & 4.3 \\
& Twdq8 & 10 & 64.5 & $45.5-66.7$ & 2.4 \\
& Twdq5 & 14 & 53.3 & $22.3-62.3$ & 2.8 \\
$\mathrm{~F}_{2} \mathrm{H}$ & Twdq2 & 1 & 68.5 & $51.5-75.5$ & 8.3 \\
& Twdq7 & 4 & 33.1 & $15.1-52.1$ & 3.3 \\
& Twdq4 & 8 & 45.5 & $15.5-58.5$ & 5.2 \\
& Twdq6 & 17 & 52.1 & $25.1-60.7$ & 5.1 \\
\hline
\end{tabular}

QTL, quantitative trait loci; $\mathrm{Cl}$, confidence interval; LOD, logarithm of the odds. 1) QTL symbols were assigned if they were significant, or if they were suggestive but identified as significant at least once previously in different genetic crosses.

2) $95 \% \mathrm{Cl}$ was defined by a 1.5-LOD drop.

3) LOD scores for significant QTL are indicated in bold. 


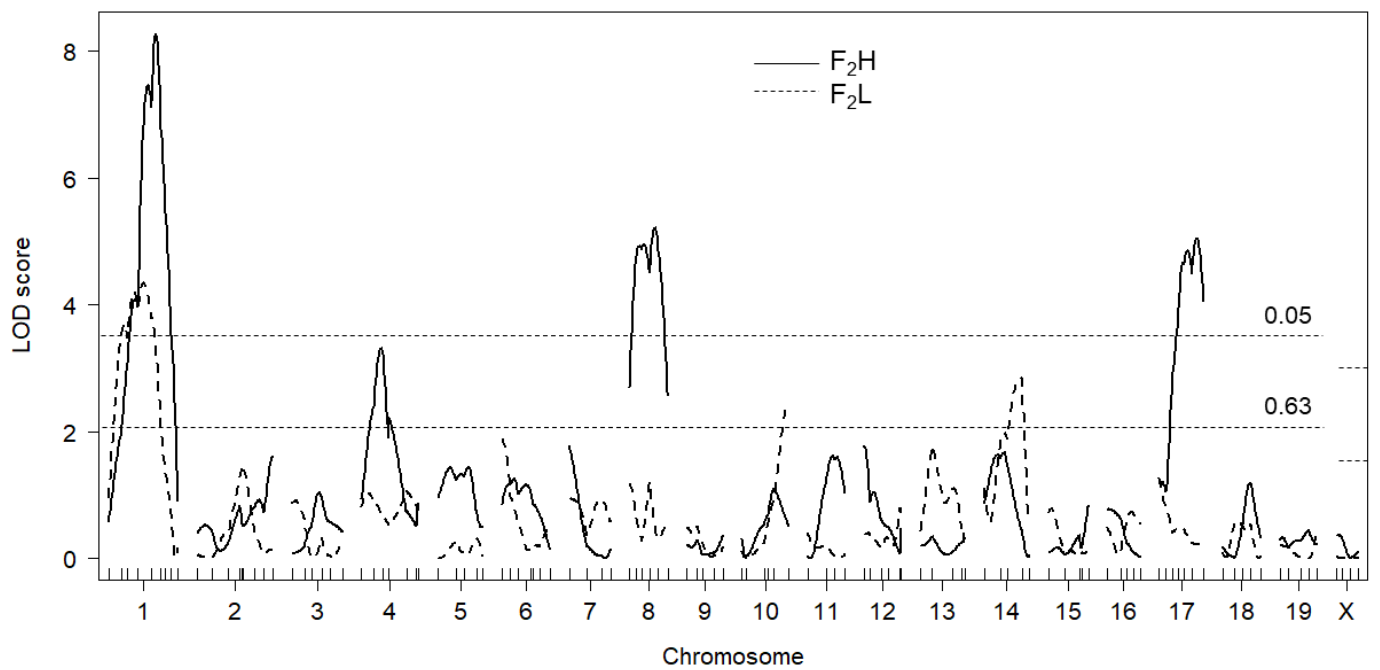

Figure 3. Genome-wide LOD score plot of the QTL for testis weight when the $\mathrm{F}_{2} \mathrm{H}$ (solid lines) and $\mathrm{F}_{2} \mathrm{~L}$ (dashed lines) mice were analyzed separately. The $x$-axis represents the chromosomal and microsatellite marker position, and the y-axis represents the LOD score. Horizontal broken lines indicate the genome-wide threshold LOD score for significant $(p<0.05)$ and suggestive $(p<0.63)$ linkage. Threshold LOD scores for significant and suggestive QTL were 3.37 and 2.08 for autosomes and 2.72 and 1.50 for Chr $X$, respectively. LOD, logarithm of the odds; QTL, quantitative trait loci.
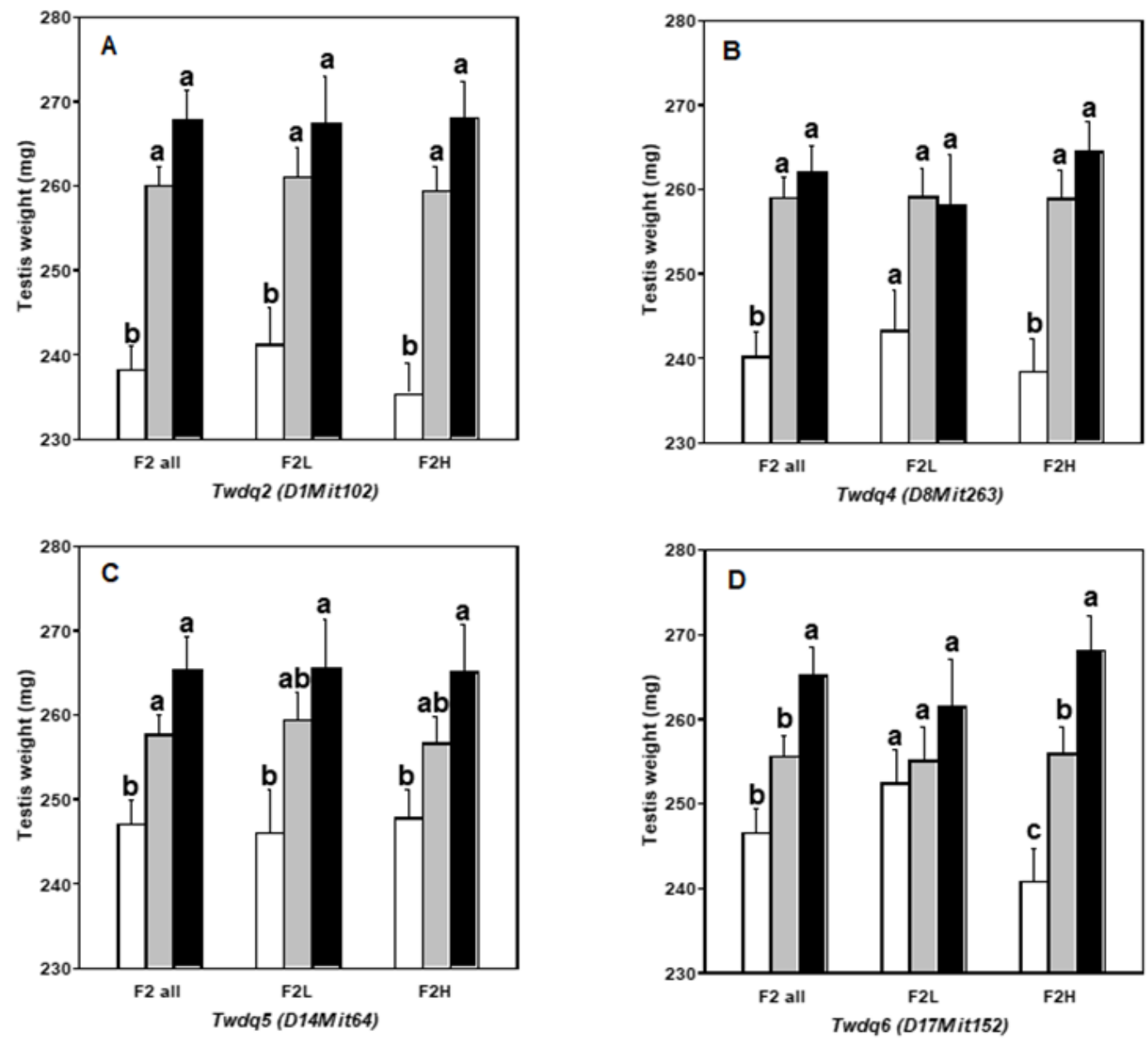

Figure 4. Comparison of allele effect of significant QTL among all $F_{2}, F_{2} L$, and $F_{2} H$ mice. The $x$-axis represents the genotype at the microsatellite marker nearest QTL, and the $y$-axis represents average testis weight. Mice with different superscripts denote the presence of significant difference. Statistical difference between mice with three genotypes within each $\mathrm{F}_{2}$ group (i.e., $\mathrm{F}_{2}$ all, $\mathrm{F}_{2} \mathrm{~L}$, and $\mathrm{F}_{2} \mathrm{H}$ ) was identified by Tukey-Kramer honestly significant difference tests. Error bars indicate standard error of means. QTL, quantitative trait loci. 
Table 4. Candidate genes, mutant phenotypes, and nsSNV identified for candidate genes of significant QTL

\begin{tabular}{|c|c|c|c|c|c|c|c|}
\hline $\mathrm{Chr}$ & Position cM & Gene & Testis weight in mutants & Location & Nucleotide change & Amino acid change & $\mathrm{db}$ SNP ID \\
\hline \multirow[t]{11}{*}{1} & 57.91 & Kiss1 & Decrease & - & - & - & - \\
\hline & 57.94 & Snrpe & Decrease & - & - & - & - \\
\hline & 58.24 & Ptprv & Increase & - & - & - & - \\
\hline & 61.45 & Aspm & Decrease & - & - & - & - \\
\hline & 67.71 & Tdrd5 & Decrease & Chr 1: 156262881 & c. $2594 C>G$ & p.Pro865Arg & rs49650703 \\
\hline & & & & Chr 1: 156263413 & c. $2495 C>T$ & p.Ala832Val & rs13476193 \\
\hline & & & & Chr 1: 156267355 & c. $2177 G>A$ & p.Gly726Glu & rs48483855 \\
\hline & & & & Chr 1: 156270646 & c. $2080 G>A$ & p.Asp694Asn & rs30769841 \\
\hline & & & & Chr 1: 156285523 & c. $1186 A>G$ & p.Thr396Ala & rs31654838 \\
\hline & & & & Chr 1: 156301806 & c. $333 A>G$ & p.lle111Met & rs32356404 \\
\hline & 71.56 & Slc19a2 & Decrease & - & - & - & - \\
\hline \multirow[t]{6}{*}{8} & 18.75 & $\operatorname{Nrg} 1$ & Decrease & Chr 8: 31818081 & c. $2050 T>A$ & p.Leu684lle & rs32559738 \\
\hline & 20.59 & Tex15 & Decrease & - & - & - & - \\
\hline & 23.89 & Cnot7 & Decrease & - & - & - & - \\
\hline & 26.87 & $\operatorname{lng} 2$ & Decrease & - & - & - & - \\
\hline & 40.45 & Nanos3 & Decrease & Chr 8: 84176519 & c. $13 A>G$ & p.Asn5Asp & rs38027221 \\
\hline & 41.25 & Syce2 & Decrease & Chr 8: 848722541 & c. $13 \mathrm{G}>\mathrm{A}$ & p.Gly5Arg & rs32744209 \\
\hline \multirow[t]{7}{*}{14} & 18.79 & $T k t$ & Decrease & - & - & - & - \\
\hline & 25.36 & $0 t \times 2$ & Decrease & - & - & - & - \\
\hline & 27.98 & $B C / 2 / 2$ & Decrease & - & - & - & - \\
\hline & 36.32 & Piwil2 & Decrease & - & - & - & - \\
\hline & 37.62 & Fndc3a & Decrease & - & - & - & - \\
\hline & 50.09 & $T b c 1 d 4$ & Increase & Chr 14: 101458822 & c. $2500 A>G$ & p.lle834Val & rs48744612 \\
\hline & & & & Chr 14: 101477059 & c. $1975 A>G$ & p.Arg659Gly & rs235588405 \\
\hline \multirow[t]{10}{*}{17} & 18.57 & Msh5 & Decrease & Chr 17: 35028832 & c. $2411 C>A$ & p.Thr804Lys & rs33561826 \\
\hline & & & & Chr 17: 35031264 & c. $1693 \mathrm{~A}>\mathrm{G}$ & p.Met565Val & rs50143709 \\
\hline & 18.59 & Tnf & Decrease & - & - & - & - \\
\hline & 18.75 & Atat1 & Decrease & Chr 17: 35909951 & c. $85 \mathrm{C}>\mathrm{T}$ & p.Pro29Ser & - \\
\hline & 25.86 & Dazl & Decrease & - & - & - & - \\
\hline & 29.4 & Safb & Decrease & - & - & - & - \\
\hline & 58.35 & Lhcgr & Decrease & - & - & - & - \\
\hline & 58.76 & Fshr & Decrease & Chr 17: 88985240 & c. $2009 C>A$ & p.Pro670His & rs107828637 \\
\hline & & & & Chr 17: 88985243 & c. $2006 A>G$ & p.Asn669Ser & rs108743480 \\
\hline & & & & Chr 17: 88986290 & C.959G $>A$ & p.Ser320Asn & rs51040992 \\
\hline
\end{tabular}

nsSNV, nonsynonymous single-nucleotide variation; QTL, quantitative trait loci; SNP, single nucleotide polymorphism; Kiss1, KiSS-1 metastasis-suppressor; Snrpe, small nuclear ribonucleoprotein E; Ptprv, protein tyrosine phosphatase, receptor type, V; Aspm, abnormal spindle microtubule assembly; Tdrd5, tudor domain containing 5; SIc19a2, solute carrier family 19 (thiamine transporter), member 2; Nrg1, neuregulin 1; Tex15, testis expressed gene 15; Cnot7, CCR4-NOT transcription complex, subunit 7; Ing2, inhibitor of growth family, member 2; Nanos3, nanos C2HC-type zinc finger 3; Syce2, synaptonemal complex central element protein 2; Tkt, transketolase; Otx2, orthodenticle homeobox 2; BCI2I2, BCL-like 2; Piwil2, piwi-like RNA-mediated gene silencing 2; Fndc3a, fibronectin type III domain containing 3A; Tbc1d4, TBC1 domain family, member 4; Msh5, mutS homolog 5; Tnf, tumor necrosis factor; Atat1, alpha tubulin acetyltransferase 1; Dazl, deleted in azoospermia-like; Safb, scaffold attachment factor B; Lhcgr, luteinizing hormone/choriogonadotropin receptor; Fshr, follicle stimulating hormone receptor.

Atat1, and follicle stimulating hormone receptor (Fshr), but none of them except for one that detected for Atat 1 were DDD-specific. p.Pro29Ser resulting from a c.85C $>$ T substitution (Chr 17:35909951) in Atat1 seems to be novel because no single nucleotide polymorphism (SNP) ID has been assigned, and because no SNP were known at this chromosomal location in other inbred mouse strains including 129S1/SvImJ,

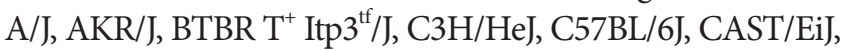
CBA/J, DBA/1J, DBA/2J, FVB/NJ, I/LnJ, KK/HIJ, MOLF/EiJ, NOD/ShiLtJ, NZB/BINJ, NZO/HILtJ, PWK/PhJ, RF/J, SPRET/ EiJ, and WSB/EiJ according to "Mouse SNP retrieval utility" by Mouse Phenome Database (Figure 5A). Furthermore, Pro29 was well conserved among mammalian species including human, rat, chimpanzee, rhesus macaque, cattle, and $\operatorname{dog}$ (Figure 5B).

\section{DISCUSSION}

This study identified nine QTL, of which five were significant and four were suggestive. The result was satisfactory because the number of QTL underlying testis weight was thought to be small $[7,20]$. Seven of the nine QTL, i.e., Twdq2 (Chr 1), 
(A)

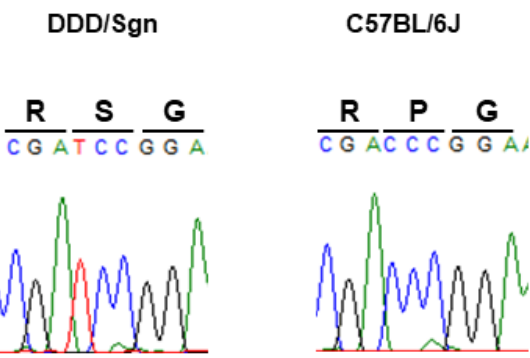

(B) Atat1 sequence

\begin{tabular}{lc} 
DDD/Sgn & \multirow{2}{*}{$* \quad 33$} \\
Rat & ARRSGTTT \\
Human & ARRPGTTT \\
Chimpanzee & ARRPGTTT \\
Rhesus macaque & ARRPGTTT \\
Cattle & ARRPGTTT \\
Dog & ARRPGTTT \\
& ARRPGTTT
\end{tabular}

Figure 5. Atat1 sequence analysis. (A) Sequencing chromatograms for Atat1 from DDD/Sgn, C57BL/6J, DH/Sgn, and CBA/N mice showing C>T polymorphism. (B) Atat1 sequence alignment from various species with the novel polymorphism in red. Atat1, alpha tubulin acetyltransferase 1.

Twdq7 (Chr 4), Twdq4 (Chr 8), Twdq5 (Chr 14), Twdq6 (Chr 17), and the suggestive QTL on Chrs 5 and 18, had also been identified in either of our preceding studies using DDD mice $[9,10]$. Therefore, only Twdq9 (Chr 7) and Twdq8 (Chr 10) were novel in a series of QTL mapping studies using DDD mice. Based on the results of three studies, most of the QTL underlying high testis weight in DDD mice might have been identified. Notably, the DDD-derived allele was associated with higher testis weight at most QTL.

The lineage effect was observed in the $F_{1}$ populations, i.e., $B D F_{1}$ mice had significantly higher testis weight than $\mathrm{DB} \mathrm{F}_{1}$ mice. The lineage effect was also found in our previous studies; e.g., $\mathrm{DH} \times \mathrm{DDD} \mathrm{F}_{1}$ mice had significantly higher testis weight than that of the $\mathrm{DDD} \times \mathrm{DH} \mathrm{F}_{1}$ mice [9], and $\mathrm{CBA} \times \mathrm{DDD} \mathrm{F}_{1}$ mice had significantly higher testis weight than that of the $\mathrm{DDD} \times \mathrm{CBA} \mathrm{F}_{1}$ mice [10]. We attribute the lineage effect to the difference in the genes on the Chr $\mathrm{Y}$ because the $\mathrm{F}_{1}$ mice carrying $\mathrm{Y}^{\mathrm{DDD}}$ had significantly higher testis weight than the reciprocal $\mathrm{F}_{1}$ mice in all crosses. There is experimental evidence supporting the contribution of $\mathrm{Chr} \mathrm{Y}$ to testis weight in mice [21]. Our studies in Y-consomic strains clearly showed the effect of $\mathrm{Chr} Y$; Chr $\mathrm{Y}^{\mathrm{DDD}}$ produced significantly heavier testes than did $\mathrm{Chr} \mathrm{Y}^{\mathrm{B} 6}[9,10]$. Further evidence was that DDD mice had significantly heavier testis than DDD-Chr $\mathrm{Y}^{\mathrm{CBA}}$ mice (296.0 $\pm 4.4 \mathrm{mg}$ vs $252.2 \pm 3.8 \mathrm{mg}, \mathrm{p}<0.0001$ ), although the body weight did not significantly differ between the two strains (32.3 $\pm 0.4 \mathrm{~g}$ vs $31.8 \pm 0.3 \mathrm{~g}, \mathrm{p}>0.3$ ) (unpublished data). It was suggested that the native $\mathrm{Y}^{\mathrm{DDD}}$ was indispensable to sustain high testis weight in DDD mice. The effect of $\mathrm{Chr} \mathrm{Y}$ appeared to be independent of the effect of autosomes.

To control the effect of body weight, we analyzed testis weight by including body weight as an additive covariate. Accordingly, we re-analyzed previously published testis weight data in the $\mathrm{DDD} \times \mathrm{DH} \mathrm{F} \mathrm{F}_{2}$ mice [9] and $\mathrm{DDD} \times \mathrm{CBA} \mathrm{F}_{2}$ mice [10] by including body weight as an additive covariate. As a result, two of four suggestive QTL identified in $\mathrm{DDD} \times \mathrm{DH}$ $\mathrm{F}_{2}$ mice, i.e., QTL on Chrs 14 and 17, were identified as significant QTL (the maximum LOD scores for these QTL were 4.8 and 4.7 , respectively). The result suggests that these QTL may have an indirect effect on testis weight, acting through the body weight [15]. The QTL on Chr17 identified in DDD $\times$ $\mathrm{DH} \mathrm{F}_{2}$ mice might be allelic to that identified in this study because of considerable increase of LOD score after the inclusion of body weight as an additive covariate. In contrast, it was uncertain whether the QTL on Chr 14 identified in the two crosses were allelic, because the LOD score for Twdq5 identified in the present study was not substantially changed after the inclusion of body weight as an additive covariate.

Results of separate $\mathrm{F}_{2}$ analyses (i.e., $\mathrm{F}_{2} \mathrm{~L}$ and $\mathrm{F}_{2} \mathrm{H}$ mice) strongly suggested that we should take the endocrinological background of the mice into consideration when assessing the effect of genes on testis weight. As it was particularly evident for the QTL on Chr 17, "high" testosterone level altered the mode of inheritance of QTL allele. In other words, "high" testosterone level tended to be associated with increasing the effect of the DDD-derived allele and with decreasing the effect of the B6-derived allele. We considered the possible mechanism for this phenomenon to be that, testosterone level might change the magnitude of expression level of genes underlying QTL. Indeed, testosterone is known to regulate gene expression levels $[22,23]$. Thus, high testosterone might be associated with up-regulation of the DDD-derived genes and was associated with down-regulation of the B6-derived genes. Otherwise, high testosterone might sensitize the cells or cellular receptors, on which the genes underlying QTL act. In this context, we were interested in the genetic basis of plasma testosterone level. Like other blood components, testosterone level is suggested to be genetically determined. Serum testosterone level is inherited in an autosomal dominant mode in pig breeds Meishan and Landrace [22], and significant QTL was identified in White Duroc $\times$ Chinese Erhualian resource population [24]. However, the mode of inheritance of circulating testosterone level was ambiguous in mice, and we could not identify any significant QTL [12]. In part, this result is associated with the extensive variation in the plasma testos- 
terone levels in DDD mice. Since the episodic testosterone secretion is known in mice [25,26], variable testosterone level even in an inbred mouse strain might not be surprising. We should be cautious about species difference when interpreting the experimental results regarding the blood testosterone levels.

By searching MGI database, we found 25 candidate genes that potentially influence testis weight within $95 \%$ CIs for four significant single-QTL on Chrs 1, 8, 14, and 17 (Table 4). Interestingly, testis weight was decreased in most mutants of the candidate genes. Dysfunctional gene mutations tend to be associated with lower testis weight; thus, the higher testis weight of DDD mice might be a consequence of altered gene functions. We performed whole-exome sequencing in DDD mice on the assumption that the higher testis weight of DDD mice is caused by coding-region variants, which are specific to the DDD strain. The analysis identified nsSNV that differed between DDD and B6 mice in 8 of 25 candidate genes: Tdrd5, Nrg1, Nanos3, Syce2, Tbc1d4, Msh5, Atat1, and Fshr (Table 4). However, most of nsSNV identified for these genes were not DDD strain-specific. For example, although there were six nsSNVs in Tdrd5, all were also found in many other inbred mouse strains. In particular, two of six nsSNV in Tdrd5 resulting in Thr396Ala and Ile111Met were also identified in $\mathrm{NZB} / \mathrm{BINJ}$ strain, which have extremely heavy testes like DDD [3]. However, no significant testis weight QTL was identified on $\mathrm{Chr} 1$ in $\mathrm{C} 57 \mathrm{BL} / 6 \mathrm{ByJ} \times \mathrm{NZB} / \mathrm{BINJ} \mathrm{F}_{2}$ mice, suggesting that these were unlikely to be causative of Twdq2. Similarly, two nsSNVs in Tbc1d4 resulting in Ile834Val and Arg659Gly were identified in NZB/BINJ, but no significant testis weight QTL was identified on Chr 14 in abovementioned $\mathrm{F}_{2}$ mice [3]; therefore, these were unlikely to be causative of Twdq5. Although the nsSNVs in Fshr are plausible candidates underlying $T w d q 6$, these nsSNVs were also found in other inbred mouse strains including a strain with very light testes such as $\mathrm{CAST} / \mathrm{EiJ}$.

One nsSNV, Pro29Ser, identified in Atat1 appears to be novel, given that no SNP ID has yet been assigned. Accordingly, we searched for this nsSNV among the various inbred mouse strains with consequent that this nsSNV was not shared by any other strains. We then investigated Atat 1 sequence in other mammalian species and found that Pro29 was well conserved. Thus, we concluded that Ser29 was a mutation occurred specifically in DDD mice. A targeted disruption mutation in Atat1 resulted in reduction of testis weight in mice [27]. Therefore, Pro29Ser was unlikely to be associated with loss or hypofunction of this gene, if this gene is a causative of Twdq6. Although further-in-depth in vivo studies are necessary for validating the function of this mutation, this was an important finding to understand the molecular basis of mechanisms underlying high testis weight in mice. Taken together, results of the present study provide insights into genetic and endocrinological mechanisms determining testis weight in mice.

\section{CONFLICT OF INTEREST}

We certify that there is no conflict of interest with any financial organization regarding the material discussed in the manuscript.

\section{ACKNOWLEDGMENTS}

We would like to thank Editage (www.editage.jp) for English language editing. This study was supported by the NIAS Strategic Research Fund.

\section{REFERENCES}

1. Harcourt AH, Harvey PH, Larson SG, Short RV. Testis weight, body weight and breeding system in primates. Nature 1981; 293:55-7. https://doi.org/10.1038/293055a0

2. Leader-Williams N. Age-related changes in the testicular and antler cycles of reindeer, Rangifer tarandus. J Reprod Fertil 1979;57:117-26. https://doi.org/10.1530/jrf.0.0570117

3. Le Roy I, Tordjman S, Migliore-Samour D, Degrelle H, Roubertoux PL. Genetic architecture of testis and seminal vesicle weights in mice. Genetics 2001;158:333-40.

4. Mafizul Islam ABM, Hill WG, Land RB. Ovulation rate of lines of mice selected for testis weight. Genet Res 1976;27: 23-32. https://doi.org/10.1017/S0016672300016207

5. Furukawa T, Awata T, Satoh M. Selection for testis size and correlated responses of female reproductive traits in golden hamsters. Anim Sci J 2013;84:382-8. https://doi.org/10.1111/ asj. 12015

6. Land RB. The expression of female sex-limited characters in the male. Nature 1973;241:208-9. https://doi.org/10.1038/ 241208a0

7. Rocha JL, Eisen EJ, Van Vleck LD, Pomp D. A large-sample QTL study in mice: II. Body composition. Mamm Genome 2004;15:100-13. https://doi.org/10.1007/s00335-003-2308-6

8. L'Hôte D, Serres C, Laissue P, et al. Centimorgan-range onestep mapping of fertility traits using interspecific recombinant congenic mice. Genetics 2007;176:1907-21. https://doi.org/ 10.1534/genetics.107.072157

9. Suto J. Genetic dissection of testis weight in a mouse strain having an extremely large testis: major testis weight determinants are autosomal rather than Y-linked on the basis of comprehensive analyses in Y-chromosome consomic strains. Proc Jpn Acad Ser B Phys Biol Sci 2008;84:393-406. https:// doi.org/10.2183/pjab.84.393

10.Suto J. Genetic dissection of testis weight in mice: quantitative trait locus analysis using F2 intercrosses between strains with extreme testis weight, and association study using Y-consomic 
strains. Mamm Genome 2011;22:648-60. https://doi.org/10. 1007/s00335-011-9353-3

11. Goto N, Nakajima Y, Imamura K, Yoshida T. Influence of testosterone on hydronephrosis in the inbred mouse strain DDD. Lab Anim 1985;19:85-8. https://doi.org/10.1258/00236 7785780942651

12. Suto J, Kojima M. Quantitative trait loci that control body weight in DDD/Sgn and C57BL/6J inbred mice. Mamm Genome 2017;28:13-9. https://doi.org/10.1007/s00335-0169666-3

13. O'Shaughnessy PJ, Monteiro A, Abel M. Testicular development in mice lacking receptors for follicle stimulating hormone and androgen. PLoS One 2012;7:e35136. https://doi.org/10. 1371/journal.pone.0035136

14. Koskenniemi JJ, Virtanen HE, Toppari J. Testicular growth and development in puberty. Curr Opin Endocrinol Diabetes Obes 2017;24:215-24. https://doi.org/10.1097/MED.0000 000000000339

15. Broman KW, Sen S. A guide to QTL mapping with R/qtl. New York, USA: Springer; 2009.

16. Broman KW, Wu H, Sen Ś, Churchill GA. R/qtl: QTL mapping in experimental crosses. Bioinformatics 2003;19:889-90. https:// doi.org/10.1093/bioinformatics/btg112

17. Churchill GA, Doerge RW. Empirical threshold values for quantitative trait mapping. Genetics 1994;138:963-71.

18.Lander E, Kruglyak L. Genetic dissection of complex traits: guidelines for interpreting and reporting linkage results. Nat Genet 1995;11:241-7. https://doi.org/10.1038/ng1195-241

19. Sen Ś, Churchill GA. A statistical framework for quantitative trait mapping. Genetics 2001; 159:371-87.

20. Chubb C. Genes regulating testis size. Biol Reprod 1992;47: 29-36. https://doi.org/10.1095/biolreprod47.1.29

21. Hayward P, Shire JGM. Y chromosome effect on adult testis size. Nature 1974;250:499-500. https://doi.org/10.1038/ 250499a0

22. Kojima M, Degawa M. Serum androgen level is determined by autosomal dominant inheritance and regulates sex-related CYP genes in pigs. Biochem Biophys Res Commun 2013;430: 833-8. https://doi.org/10.1016/j.bbrc.2012.11.060

23. Kojima M, Sekimoto M, Degawa M. Androgen-mediated down-regulation of CYP1A subfamily genes in the pig liver. J Endocrinol 2010;207:203-11. https://doi.org/10.1677/JOE-100160

24. Ren DR, Ren J, Xing YY, et al. A genome scan for quantitative trait loci affecting male reproductive traits in a White Duroc $\times$ Chinese Erhualian resource population. J Anim Sci 2009; 87:17-23. https://doi.org/10.2527/jas.2008-0923

25. Bartke A, Dalterio S. Evidence for episodic secretion of testosterone in laboratory mice. Steroids 1975;26:749-56. https:// doi.org/10.1016/0039-128X(75)90107-5

26. Klomberg KF, Garland, Jr T, Swallow JG, Carter PA. Dominance, plasma testosterone levels, and testis size in house mice artificially selected for high activity levels. Physiol Behav 2002;77: 27-38. https://doi.org/10.1016/S0031-9384(02)00767-9

27. Kalebic N, Sorrentino S, Perlas E, Bolasco G, Martinez C, Heppenstall PA. alphaTAT1 is the major alpha-tubulin acetyltransferase in mice. Nat Commun 2013;4:1962. https://doi. org/10.1038/ncomms2962 